\title{
COMPROMISO PARAMETRIAL EN PACIENTES CON CÁNCER DE CÉRVIX IB-1 TRATADAS CON HISTERECTOMÍA RADICAL EN PERÚ
}

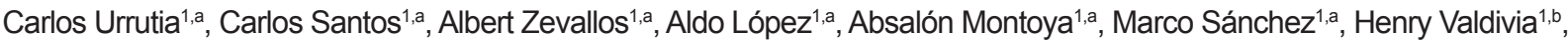 \\ Carlos Velarde ${ }^{1, a}$, Raúl Mantilla ${ }^{1, c}$, Esperanza Milla ${ }^{1, d}$, Carlos Castañeda ${ }^{1, e}$, Mayer Zaharia ${ }^{1, f}$, Manuel Álvarez ${ }^{1, g}$
}

\begin{abstract}
RESUMEN
Objetivos. Evaluar la frecuencia y factores asociados al compromiso parametrial $(\mathrm{CP})$ en pacientes con cáncer de cérvix IB-1 sometidas a histerectomía radical (HR) en el Instituto Nacional de Enfermedades Neoplásicas del Perú. Materiales y métodos. Estudio transversal analítico en 214 pacientes con cáncer de cérvix IB-1 sometidas a HR con linfadenectomía pélvica entre el 2007 y 2012. Se comparó la población con y sin CP según variables clinicopatológicas como edad, tamaño tumoral, profundidad de infiltración, grado histológico y afectación ganglionar pélvica. Resultados. Diez pacientes $(4,6 \%)$ presentaron CP. El análisis multivariable mostró que la profundidad de la invasión (OR 8,37, IC 95\%: 1,24-56,41, p=0,029) y el compromiso de ganglios pélvicos (OR 18,03, IC 95\%: 3,30-98,44, $p=0,001$ ) resultaron ser predictores independientes del CP. La presencia de tamaño tumoral $\leq 2 \mathrm{~cm}$, invasión $\leq 10 \mathrm{~mm}$, ausencia de permeación linfovascular y ausencia de compromiso ganglionar permitió identificar 97 pacientes de bajo riesgo de $\mathrm{CP}$, de los cuales ninguno tuvo CP en patología. Conclusiones. Confirmamos la baja frecuencia del CP en el subgrupo de cáncer de cervix IB-1 y su asociación con la profundidad de la invasión y con el compromiso ganglionar linfático sugeridos en estudios previos. Esta información permitirá identificar un subgrupo de pacientes con bajo riesgo de CP candidatas a una cirugía menos radical en nuestra institución.
\end{abstract}

Palabras clave: Cáncer del cuello uterino; Histerectomía; Escisión del ganglio linfático (fuente: DeCS BIREME).

\section{PARAMETRIAL INVOLVEMENT IN CERVICAL CANCER IB1 PATIENTS TREATED WITH RADICAL HYSTERECTOMY IN PERU}

\begin{abstract}
Objectives. To assess the frequency and factors associated with parametrial involvement (PI) in patients with cervical cancer IB1 who underwent radical hysterectomy $(\mathrm{RH})$ at the National Institute of Neoplastic Diseases in Peru. Materials and methods. Cross-sectional study of 214 patients with cervical cancer IB1 undergoing RH with pelvic lymphadenectomy between 2007 and 2012. The population was compared with and without clinicopathological variables associated with PI such as age, tumor size, depth of infiltration, histological grade and pelvic lymph node involvement. Results. Ten patients (4.6\%) had PI. Multivariate analysis showed that the depth of invasion (OR 8.37, 95\% Cl 1.24- 56.41, $\mathrm{p}=0.029)$ and pelvic node involvement (OR 18.03; 95\% Cl 3.30-98.44, $p=0.001$ ) were all independent predictors of PI. The presence of tumor size $\leq 2 \mathrm{~cm}$, invasion $\leq 10 \mathrm{~mm}$, absence of lymphovascular permeation and absence of nodal involvement identified 97 patients at low risk of PI, none of whom had PI pathology. Conclusions. We confirmed the low frequency of PI in the cervical cancer IB1 subgroup and its association with the depth of invasion and lymph node involvement suggested in previous studies. This information will allow the identification of a subgroup of patients at low risk of PI for less radical surgery in our institution.
\end{abstract}

Key words: Uterine cervical neoplasms; Hysterectomy; Lymph node excision (source: MeSH NLM).

\section{INTRODUCCIÓN}

El cáncer de cérvix es el más común y la principal causa de muerte en mujeres peruanas; lo que lo convierte en un serio problema de salud pública. Los programas de detección implementados en Perú permiten identificar esta neoplasia en estadios tempranos y nos presenta el reto de realizar un manejo quirúrgico anticipado y adecuado de la enfermedad ${ }^{(1,2)}$.

Las pacientes en estadio $1 \mathrm{~A} 2$ y $1 \mathrm{~B} 1$ son habitualmente tratadas con histerectomía radical (HR) y linfadenectomía pélvica, con una supervivencia global a los 5 años que alcanza el 90 a $95 \%{ }^{(3-6)}$. El compromiso del parametrio

\footnotetext{
Instituto Nacional de Enfermedades Neoplásicas. Lima, Perú

a Cirujano oncólogo; ${ }^{\mathrm{b}}$ cirujano oncólogo, magíster en Salud Publica; ${ }^{\mathrm{c}}$ bachiller en Bioestadística; ${ }^{\mathrm{d}}$ médico patólogo; ${ }^{\mathrm{e}}$ oncólogo clínico, magíster en Genética y Biología Celular, magíster en Oncología Avanzada; ${ }^{\mathrm{f}}$ médico radioncólogo, magíster en Radioncología, doctor en Medicina; ${ }^{\mathrm{g}}$ cirujano oncólogo, magíster en Medicina.

Recibido: : 04-02-14 Aprobado: 28-05-14
}

Citar como: Urrutia C, Santos C, Zevallos A, López A, Montoya A, Sánchez M, et al. Compromiso parametrial en pacientes con cáncer de cérvix IB-1 tratadas con histerectomía radical en Perú. Rev Peru Med Exp Salud Publica. 2014;31(3):431-36. 
contiguo al cérvix representa el sitio más frecuente de diseminación local (6 a $31 \%$ en EC IB) y se produce mediante la extensión microscópica directa o mediante permeación de émbolos tumorales por vía linfovascular ${ }^{(7-10)}$. Por lo tanto, la eliminación del tejido parametrial (parametrectomía) ha sido considerada como parte del manejo quirúrgico estándar de cáncer de cérvix. Sin embargo, este procedimiento puede producir denervación parcial de las vías autonómicas a los órganos de la pelvis asociada a disfunción de la vejiga, disfunción orgásmica y trastornos de la motilidad anorrectal ${ }^{(11-13)}$. Así, Landoni et al. reportaron morbilidad tardía que incluye complicaciones urológicas y vesicales en más del $28 \%$ de 238 mujeres sometidas a HR ${ }^{(14)}$.

Algunos estudios sugieren que técnicas de imágenes actuales como la resonancia magnética tienen una precisión de hasta el 95\% en identificar el CP, sin embargo el tamaño muestral es pequeño y no se ha aceptado a esta prueba como parte estándar del estudio preoperatorio $(15,16)$. Por lo tanto, la radicalidad de la cirugía y el estudio histológico de la pieza operatoria conforman el único método definitivo de asegurar la situación y extensión de la enfermedad en el parametrio.

Las mujeres con tumores $<2 \mathrm{~cm}$, con infiltración $<10 \mathrm{~mm}$, sin invasión del espacio linfovascular y sin compromiso de los ganglios pélvicos tienen una probabilidad muy baja de CP. Así, un subgrupo de pacientes con cáncer de cérvix temprano podría ser sometido a cirugía menos radical y evitar la HR. Un estudio recientemente publicado por Rob et al. describe la factibilidad y seguridad de realizar procedimientos menos radicales y que permiten la conservación de la fertilidad ${ }^{(7,17-19)}$.

A pesar de la relevancia y las medidas sanitarias implementadas para esta neoplasia en Perú, no existe un estudio publicado con información que permita identificar a pacientes con bajo riesgo de CP candidatos a una cirugía menos radical. El presente estudio tiene como objetivo evaluar la frecuencia y factores asociados al CP en pacientes con cáncer de cérvix IB-1 sometidas a HR en el Instituto Nacional de Enfermedades Neoplásicas (INEN) del Perú.

\section{MATERIALES Y MÉTODOS}

\section{DISEÑO Y POBLACIÓN DE ESTUDIO}

Estudio observacional, transversal analítico. Se revisaron las historias clínicas y reportes de anatomía patológica de 214 pacientes con cáncer de cérvix en estadio figo IB-1 que cumplieron los criterios de inclusión: información clínico- patológica completa, haber sido sometidas HR (clase II y III) y linfadenectomía pélvica durante septiembre del 2007 y agosto de 2012, en el INEN de Lima, Perú.

\section{PROCEDIMIENTOS}

El examen macroscópico del cuerpo uterino y cérvix incluyó la evaluación del tejido parametrial e identificación de ganglios linfáticos parametriales. La profundidad de la invasión tumoral se definió como la suma de estas medidas en la biopsia de cono y la pieza de histerectomía. El CP se definió como presencia de células neoplásicas en el tejido parametrial, compromiso linfovascular o compromiso de ganglios parametriales. La permeación linfovascular positiva se definió como la presencia inequívoca de células malignas en espacios revestidos por endotelio observados en el examen histológico de los especímenes

Las pacientes fueron divididas en dos grupos de acuerdo a la presencia de CP (Figura 1). Ambos grupos
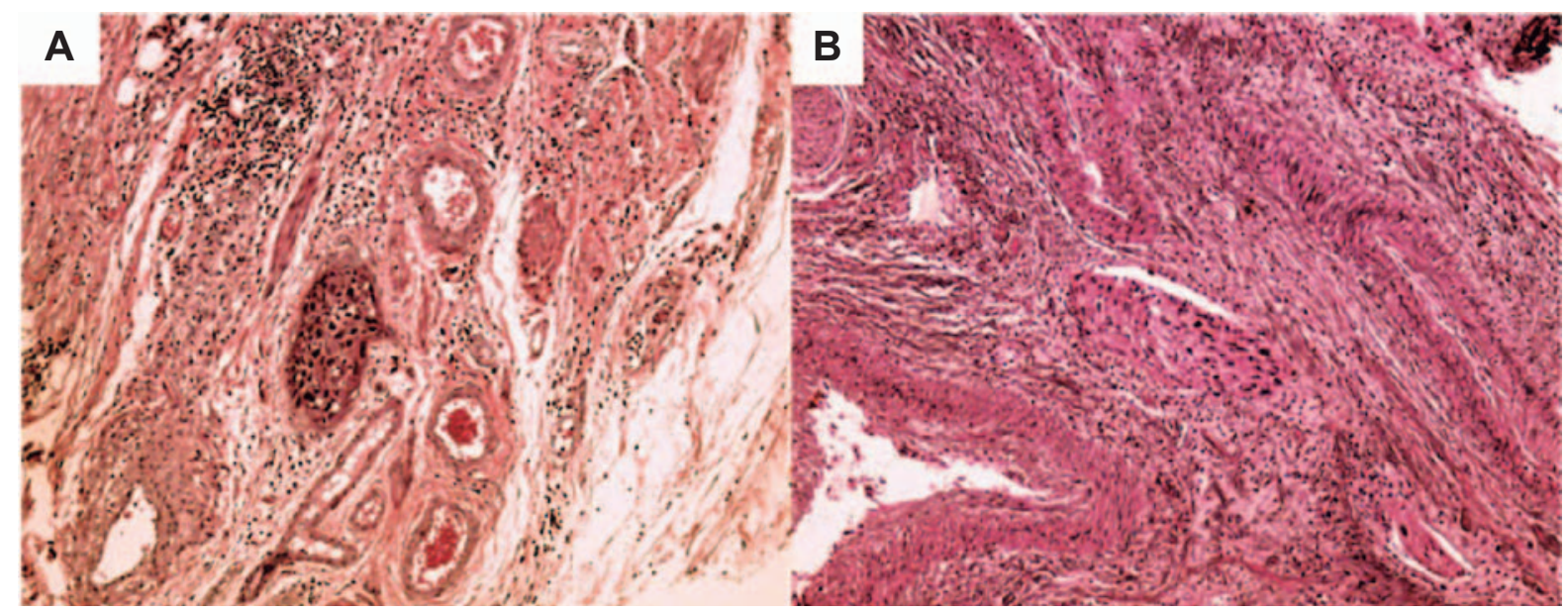

Figura 1. A) Células neoplásicas en nicho que infiltran el tejido parametrial por extensión directa. B) Émbolos tumorales dentro de la vasculatura parametrial. (HE, 40x) 
se compararon con respecto a las variables edad, tamaño tumoral, profundidad de infiltración, invasión del espacio linfovascular, grado histológico, tipo histológico y la afectación ganglionar.

\section{ANÁLISIS ESTADÍSTICO}

Las evaluaciones estadísticas incluyeron tabulaciones descriptivas de las características clínicas de los pacientes. El análisis bivariado se realizó mediante la prueba chi-cuadrado con corrección de Yates. En el análisis multivariable, el compromiso parametrial fue analizado en relación a las demás características clínicas mediante el modelo de regresión logística con selección paso a paso. Todas las evaluaciones se realizaron a un nivel de significancia de $5 \%$. El análisis estadístico se realizó con el programa SPSS 19.

\section{ASPECTOS ÉTICOS}

El desarrollo del estudio no involucró contacto ni intervención sobre los pacientes. La base de datos no incluyó la identidad de las pacientes y la información obtenida se mantuvo en completa confidencialidad. El proyecto fue aprobado por el Comité de Ética del Instituto Nacional de Enfermedades Neoplásicas del Perú.

\section{RESULTADOS}

Se encontró CP en diez $(4,7 \%)$ de los 214 pacientes con cáncer de cérvix en estadio figo IB-1 sometidas a HR. La edad promedio fue 44,3 años (rango de 21 a 69 años). El promedio del tamaño y la profundidad tumoral fueron de $19,8 \mathrm{~mm}$ y $8,3 \mathrm{~mm}$, respectivamente. El tipo histológico más frecuente fue el epidermoide en 159 pacientes $(72,3 \%)$; el grado tumoral más frecuente fue el GII en 185 pacientes (86,4\%); se registraron 57 $(26,6 \%)$ pacientes con permeación linfovascular; 25 $(11,7 \%)$ tuvieron ganglios pélvicos positivos; $22(10,3 \%)$ presentaron compromiso de cuerpo uterino y $6(2,8 \%)$ tuvieron compromiso de margen quirúrgico (Tabla 1).

La proporción de pacientes con tamaño tumoral > 20 $\mathrm{mm}$ fue mayor en el grupo de parametrio comprometido $(80,0 \%)$ que en el grupo sin compromiso parametrial $(38,2 \%) \quad(p=0,021)$. La proporción de pacientes con profundidad tumoral mayor de $10 \mathrm{~mm}$ fue mayor en el grupo con parametrio comprometido $(80,0 \%)$ que en el grupo sin compromiso parametrial $(23,5 \%)(p<0,001)$. La proporción de pacientes con grado G3 fue mayor en el grupo de parametrio comprometido $(20,0 \%)$ que en el grupo sin parametrio comprometido $(6,4 \%)(p=0,041)$. La proporción de pacientes con permeación linfovascular
Tabla 1. Características de las pacientes sometidas a histerectomía radical en el Instituto Nacional de Enfermedades Neoplásicas, Perú 2007-2012

\begin{tabular}{|c|c|c|}
\hline Características & $\mathbf{N}$ & $(\%)$ \\
\hline Edad* $^{*}$ & 44,3 & $(21-69)$ \\
\hline Tamaño tumoral $(\mathrm{mm})^{*}$ & 19,8 & $(5-40)$ \\
\hline Profundidad tumoral $(\mathrm{mm})^{*}$ & 8,3 & $(1,5-27)$ \\
\hline \multicolumn{3}{|l|}{ Histología } \\
\hline Epidermioide & 159 & $(74,3)$ \\
\hline Adenocarcinoma & 49 & $(22,9)$ \\
\hline Adenoescamoso & 6 & $(2,8)$ \\
\hline \multicolumn{3}{|l|}{ Grado tumoral } \\
\hline G1 & 13 & $(6,1)$ \\
\hline G2 & 185 & $(86,4)$ \\
\hline G3 & 16 & $(7,5)$ \\
\hline \multicolumn{3}{|c|}{ Permeación linfovascular positiva } \\
\hline Sí & 57 & $(26,6)$ \\
\hline No & 157 & $(73,4)$ \\
\hline \multicolumn{3}{|c|}{ Compromiso ganglionar pélvico } \\
\hline Sí & 25 & $(11,7)$ \\
\hline No & 189 & $(88,3)$ \\
\hline \multicolumn{3}{|l|}{ Parametrio comprometido } \\
\hline Sí & 10 & $(4,7)$ \\
\hline No & 204 & $(95,3)$ \\
\hline \multicolumn{3}{|l|}{ Compromiso uterino } \\
\hline Sí & 22 & $(10,3)$ \\
\hline No & 192 & $(89,7)$ \\
\hline \multicolumn{3}{|c|}{ Margen quirúrgico comprometido } \\
\hline Sí & 6 & $(2,8)$ \\
\hline No & 208 & $(97,2)$ \\
\hline
\end{tabular}

positiva fue mayor en el grupo con parametrio comprometido $(80,0 \%)$ que en el grupo sin parametrio comprometido $(24,0 \%) \quad(p<0,001)$. La proporción de pacientes con ganglios pélvicos positivos fue mayor en el grupo con parametrio comprometido $(70,0 \%)$ que en el grupo sin compromiso parametrial $(8,8 \%)(p<0,001)$. La edad, el tipo histológico, el compromiso del útero y el margen quirúrgico no presentaron asociación con $\mathrm{CP}$. (Tabla 2)

En el análisis multivariable se encontró como predictores independientes el tener una profundidad tumoral mayor de $10 \mathrm{~mm}$ (OR 8,37, IC 95\%: 1,24-56,41, p=0,029) con respecto a tener una profundidad $\leq 10 \mathrm{~mm}$, y tener compromiso ganglionar pélvico (OR 18,03, IC 95\%: $3,30-98,44, p=0,001)$ con respecto a la ausencia de compromiso ganglionar pélvico (Tabla 3 ).

La aplicación de la combinación de las siguientes características tumorales: tamaño $<20 \mathrm{~mm}$, profundidad tumoral $<10 \mathrm{~mm}$, ausencia de permeación linfovascular y ausencia de compromiso ganglionar en la totalidad 
Tabla 2. Características asociadas con compromiso parametrial en pacientes sometidas a histerectomía radical en el Instituto Nacional de Enfermedades Neoplásicas, 2007-2012

\begin{tabular}{|c|c|c|c|}
\hline \multirow[t]{2}{*}{ Características } & \multicolumn{2}{|c|}{$\begin{array}{l}\text { Parametrio } \\
\text { comprometido }\end{array}$} & \multirow{2}{*}{$\begin{array}{c}\text { Valor } \\
p\end{array}$} \\
\hline & Sí (\%) & No (\%) & \\
\hline \multicolumn{4}{|l|}{ Edad } \\
\hline 50 o menos años & $6(60,0)$ & $147(72,1)$ & 0,641 \\
\hline Más de 50 años & $4(40,0)$ & $57(27,9)$ & \\
\hline \multicolumn{4}{|l|}{ Tamaño tumoral (mm) } \\
\hline 20 o menos & $2(20,0)$ & $126(61,8)$ & 0,021 \\
\hline Más de 20 & $8(80,0)$ & $78(38,2)$ & \\
\hline \multicolumn{4}{|c|}{ Profundidad tumoral (mm) } \\
\hline 10 o menos & $2(20,0)$ & $156(76,5)$ & $<0,001$ \\
\hline Más de 10 & $8(80,0)$ & $48(23,5)$ & \\
\hline \multicolumn{4}{|l|}{ Histología } \\
\hline Epidermioide & $8(80,0)$ & $151(74,0)$ & 0,959 \\
\hline Otros & $2(20,0)$ & $53(26,0)$ & \\
\hline \multicolumn{4}{|l|}{ Grado tumoral } \\
\hline G1-G2 & $7(70,0)$ & $191(93,6)$ & 0,041 \\
\hline G3 & $3(30,0)$ & $13(6,4)$ & \\
\hline \multicolumn{4}{|c|}{ Permeación linfovascular positiva } \\
\hline Sí & $8(80,0)$ & $49(24,0)$ & $<0,001$ \\
\hline No & $2(20,0)$ & $155(76,0)$ & \\
\hline \multicolumn{4}{|c|}{ Compromiso ganglionar pélvico } \\
\hline Sí & $7(70,0)$ & $18(8,8)$ & $<0,001$ \\
\hline No & $3(30,0)$ & $186(91,2)$ & \\
\hline \multicolumn{4}{|l|}{ Compromiso uterino } \\
\hline Sí & $3(30,0)$ & $19(9,3)$ & 0,116 \\
\hline No & $7(70,0)$ & $185(90,7)$ & \\
\hline \multicolumn{4}{|c|}{ Margen quirúrgico comprometido } \\
\hline Sí & $1(10,0)$ & $5(2,5)$ & 0,667 \\
\hline No & $9(90,0)$ & $199(97,5)$ & \\
\hline
\end{tabular}

de pacientes incluidos en nuestra serie (214 casos) identifica 97 pacientes con estos criterios (asociados a bajo riesgo de CP en la bibliografía). Ninguno de ellos presentó compromiso tumoral en el estudio anatomopatológico.

Tabla 3. Análisis multivariable para el riesgo de compromiso parametrial en pacientes sometidas a histerectomía radical en el Instituto Nacional de Enfermedades Neoplásicas, Perú 2007-2012

\begin{tabular}{|c|c|c|}
\hline & OR (IC 95\%) & Valor $p$ \\
\hline \multicolumn{3}{|c|}{ Profundidad tumoral (mm) } \\
\hline 10 o menos & 1,00 & \\
\hline Más de 10 & $8,37(1,24-56,41)$ & 0,029 \\
\hline \multicolumn{3}{|c|}{ Compromiso ganglionar pélvico } \\
\hline No & 1,00 & \\
\hline Sí & $18,03(3,30-98,44)$ & 0,001 \\
\hline
\end{tabular}

\section{DISCUSIÓN}

En la serie de pacientes la frecuencia de $\mathrm{CP}$ en cáncer de cérvix estadio IB-1 es del 4,7\%. Este valor está dentro del rango reportado por otras investigaciones en poblaciones similares (que son del 2,4 al 10,9\% (18,20,21)). Lo que implica que se podría realizar en forma segura un procedimiento quirúrgico menos radical en un subgrupo de estas pacientes. Asimismo, la asociación entre CP y profundidad de invasión, tamaño tumoral, grado tumoral, permeación linfovascular y ganglios pélvicos positivos, también se encuentran de acuerdo con otros reportes. Se encontró que el estado ganglionar linfático pélvico es el predictor independiente más fuerte para presentar $\mathrm{CP}$, conjuntamente con la profundidad de infiltración. Este factor ha sido previamente reportado como un predictor sólido de propagación parametrial ${ }^{(7,18,21-28)}$.

Los datos hallados no muestran asociación entre la edad y el CP, relación que sí es descrita por Covens et al. en una cohorte de 842 mujeres. Sin embargo, la edad media de las mujeres incluidas en su serie con afectación del parametrio fue de 42 años, y la edad media de las personas sin participación parametrial fue de 40 años. La importancia clínica de una diferencia de tan solo dos años de edad, aun cuando alcance la significancia estadística, probablemente sea insignificante ${ }^{(7)}$.

Tampoco se encontró asociación entre el subtipo histológico y la probabilidad de tener CP, en comparación, Wright et al. reportaron una relación entre la histología y el CP en una serie de 594 mujeres donde el 3\% de 135 casos con adenocarcinoma tuvo participación parametrial en comparación con el $12 \%$ de los 401 epidermoide y un $26 \%$ de las 53 lesiones clasificadas como "otros". Sin embargo, no está claro si el subgrupo de tumores que fueron clasificados como "otros" incluyó histología de alto riesgo como carcinoma de células claras, melanoma o tumores neuroendocrinos ${ }^{(21)}$. No se encontró estas últimas histologías en esta serie, pero sí se halló tumores adenoescamosos, una histología más agresiva que los adenocarcinomas o lesiones epidermoides ${ }^{(26)}$.

Para reducir la morbilidad posoperatoria asociada con la $\mathrm{HR}$, el reto es identificar un subgrupo de pacientes con bajo riesgo de CP en quienes la parametrectomía se podría evitar sin reducir la eficacia del procedimiento. Existen varios estudios que definen este subgrupo de bajo riesgo, la Tabla 4 resume los resultados de la revisión de la literatura. La mayoría de los estudios coinciden en que el tamaño tumoral $<2 \mathrm{~cm}$ es un criterio de bajo riesgo. Algunos estudios coinciden en otros criterios de bajo riesgo que alcanzan significancia estadística, como la ausencia de permeación linfovascular, la ausencia de compromiso de ganglios linfáticos de la pelvis, o la profundidad de la invasión menor a $10 \mathrm{~mm}{ }^{(7,18,21-23)}$. 
Tabla 4. Estudios de evaluación de los factores predictivos de afectación del parametrio, criterios de bajo riesgo

\begin{tabular}{|c|c|c|c|c|c|c|c|c|c|c|}
\hline \multirow{2}{*}{ Estudio } & \multirow{2}{*}{ País } & \multirow{2}{*}{ Año } & \multirow{2}{*}{$\mathbf{N}$} & \multirow{2}{*}{$\begin{array}{l}\text { N-Bajo } \\
\text { Riesgo }\end{array}$} & \multirow{2}{*}{$\begin{array}{c}\text { Tamaño } \\
(\mathrm{mm})\end{array}$} & \multirow{2}{*}{$\begin{array}{l}\text { Invasión } \\
\text { (mm) }\end{array}$} & \multirow{2}{*}{$\begin{array}{l}\text { Permeación } \\
\text { linfovascular }\end{array}$} & \multirow{2}{*}{$\begin{array}{c}\text { Compromiso } \\
\text { ganglionar }\end{array}$} & \multicolumn{2}{|c|}{$\begin{array}{c}\text { Compromiso } \\
\text { parametrial }\end{array}$} \\
\hline & & & & & & & & & Global & Bajo riesgo \\
\hline Kinney ${ }^{(23)}$ & EE. UU. & 1995 & - & 83 & $<20$ & - & Negativo & - & - & $0 \%$ \\
\hline Covens ${ }^{(11)}$ & Canada & 2002 & 842 & 536 & $<20$ & $<10$ & - & Negativo & $4 \%$ & $0,6 \%$ \\
\hline Wright ${ }^{(23)}$ & EE. UU. & 2007 & 594 & 270 & $<20$ & - & Negativo & Negativo & $10,8 \%$ & $0,4 \%$ \\
\hline Stegeman ${ }^{(28)}$ & Países Bajos & 2007 & - & 103 & $<20$ & $<10$ & - & Negativo & - & $1,9 \%$ \\
\hline Frumovitzl (17) & EE. UU. & 2009 & 350 & 125 & $<20$ & - & Negativo & - & $7,7 \%$ & $0 \%$ \\
\hline Kodama $^{(25)}$ & Japón & 2011 & 200 & 68 & IB-1 & $<10$ & Negativo & - & $10 \%$ & $0 \%$ \\
\hline Jung ${ }^{(10)}$ & Corea & 2012 & 84 & 46 & $<10$ & - & - & - & $2,4 \%$ & $0 \%$ \\
\hline Chang (25) & Corea & 2012 & 317 & 185 & $\leq 30$ & - & - & - & $5,4 \%$ & $1,1 \%$ \\
\hline Gemer ${ }^{(30)}$ & Israel & 2013 & 530 & 107 & $\leq 20$ & - & Negativo & Negativo & $10,9 \%$ & $0 \%$ \\
\hline INEN & Perú & 2013 & 214 & 97 & $\leq 20$ en IB-1 & $\leq 10$ & Negativo & Negativo & $4.7 \%$ & $0 \%$ \\
\hline
\end{tabular}

El análisis de validación de firma clínica de factores de bajo riesgo de CP: tamaño $\leq 2 \mathrm{~cm}$, invasión $\leq 10 \mathrm{~mm}$, permeación linfovascular negativa y sin compromiso ganglionar a la población analizada identifica 97 pacientes que cumplen estas características, y ninguna de ellas presenta $\mathrm{CP}$ en el estudio patológico. La revisión bibliográfica encuentra una frecuencia global de CP en esta población de pacientes de bajo riesgo del 0,4\% (8/1977), lo que indica que la combinación de variables clínico-patológicas puede identificar pacientes con bajo riesgo de CP candidatas a cirugía que no requieren extirpación de parametrio. A pesar de que estos criterios son basados en características patológicas luego de cirugía, la biopsia cono podría identificar el tamaño y profundidad tumoral y el estado de la permeación linfovascular. Recientes estudios sugieren que los dos primeros factores pueden ser también evaluados por métodos de imágenes como RMN ${ }^{(29)}$.

Como limitaciones, si bien el tamaño muestral es reducido y la metodología utilizada fue retrospectiva para realizar análisis a profundidad, los resultados son cercanos a lo reportado por autores extranjeros, y la información obtenida podría ser incluida en futuras revisiones sistemáticas o metaanálisis para obtener conclusiones más sólidas. Así mismo, la información presentada se complementara con la futura información que se obtendrá de ensayos clínicos en actual seguimiento (ClinicalTrials.gov identifier: NCT00506376).
En conclusión este estudio confirma la baja frecuencia de CP en el subgrupo de bajo riesgo de cáncer de cérvix IB-1 y la asociación directa del CP con el estado de los ganglios linfáticos e infiltración $\leq 10 \mathrm{~mm}$ sugeridas en estudios previos. De confirmarse estos resultados en estudios multicéntricos randomizados, la cirugía menos radical, como la histerectomía simple, taquelectomía simple, o la conización con linfadenectomía pélvica, pueden ser opciones terapéuticas razonables para mujeres con tumores que cumplan criterios de bajo riesgo. La tendencia actual es a subclasificar entidades clínicas para administrar tratamientos personalizados y así la cirugía oncológica debe planificarse a medida de las necesidades de las pacientes, equilibrando la morbilidad sin perder seguridad oncológica.

Contribuciones de autoría: CUR, MAL, HVF y EMN participaron en la concepción y diseño del artículo; CUR y MSS participaron en la recolección y obtención de resultados; CUR participo en el Análisis e interpretación de datos; AZC, ALB y AMG participaron en la redacción del artículo; CSO, MZB y CAC participaron en la revisión crítica del artículo; CUR, MAL y HVF realizaron la aprobación de su versión final; CUR obtuvo el financiamiento; RMQ participó en la asesoría estadística.

Fuentes de financiamiento: autofinanciado.

Conflictos de interés: los autores declaran no tener conflictos de interés.

\section{REFERENCIAS BIBLIOGRÁFICAS}

1. Hakama M, Louhivuori K. A screening programme for cervical cancer that worked. Cancer surv. 1988;7(3):403-16.

2. Centro de Investigacion en Cancer Maes Heller INEN. Registro de cáncer de Lima Metropolitana 1994-1997. Lima: INEN; 2004.

3. Benedet JL, Odicino F, Maisonneuve P, Beller U, Creasman WT, Heintz AP, et al. Carcinoma of the cervix uteri. J Epidemiol Biostat. 2001;6(1):7-43.

4. Landoni F, Maneo A, Colombo A, Placa F, Milani R, Perego P, et al. Randomised study of radical surgery versus radiotherapy for stage Ib-IIa cervical cancer. Lancet. Aug 23;350(9077):535-40.

5. Frigerio L, Busci L, Rabaiotti E, Mariani A. Adjunctive radiotherapy after radical hysterectomy in high risk early stage cervical carcinoma. Assessment of morbidity and recurrences. Eur J Gynaecol Oncol. 1994;15(2):132-7.

6. Fiorica JV, Roberts WS, Greenberg H, Hoffman MS, LaPolla JP, Cavanagh D, et al. Morbidity and survival patterns in patients after radical hysterectomy and postoperative adjuvant pelvic 
radiotherapy. Gynecol Oncol. 1990 Mar;36(3):343-7.

7. Covens A, Rosen B, Murphy J, Laframboise $S$, DePetrillo AD, Lickrish G, et al. How important is removal of the parametrium at surgery for carcinoma of the cervix?. Gynecol Oncol. 2002 Jan;84(1):145-9.

8. Benedetti-Panici P, Maneschi F, D’Andrea G, Cutillo G, Rabitti $\mathrm{C}$, Congiu $\mathrm{M}$, et al. Early cervical carcinoma: the natural history of lymph node involvement redefined on the basis of thorough parametrectomy and giant section study. Cancer. 2000 May 15;88(10):2267-74.

9. Delgado G, Bundy BN, Fowler WC Jr, Stehman FB, Sevin B, Creasman WT, et al. A prospective surgical pathological study of stage I squamous carcinoma of the cervix: a Gynecologic Oncology Group Study. Gynecol Oncol. 1989 Dec;35(3):314-20.

10. Landoni F, Bocciolone L, Perego P, Maneo A, Bratina G, Mangioni C. Cancer of the cervix, FIGO stages IB and IIA: patterns of local growth and paracervical extension. Int J Gynecol Cancer. 1995 Sep;5(5):329-34.

11. Rob L, Halaska M, Robova H. Nervesparing and individually tailored surgery for cervical cancer. Lancet Oncol. 2010 Mar;11(3):292-301. doi: 10.1016/S1470-2045(09)70191-3.

12. Altgassen C, Hertel H, Brandstädt A, Köhler C, Dürst M, Schneider A, et al. Multicenter validation study of the sentinel lymph node concept in cervical cancer: AGO Study Group. J Clin Oncol. 2008 Jun 20;26(18):2943-51. doi: 10.1200/JCO.2007.13.8933.

13. Cai HB, Chen HZ, Zhou YF, Lie DM, Hou HY. Class II radical hysterectomy in low-risk IB squamous cell carcinoma of cervix: a safe and effective option. Int J Gynecol Cancer. 2009 Jan;19(1):46-9. doi: 10.1111/ IGC.0b013e318197f847.

14. Landoni F, Maneo A, Cormio G, Perego P, Milani R, Caruso $\mathrm{O}$, et al. Class II versus class III radical hysterectomy in stage IB-IIA cervical cancer: a prospective randomized study. Gynecol Oncol. 2001 Jan;80(1):3-12.

15. Wagenaar HC, Trimbos JB, Postema S, Anastasopoulou A, van der Geest RJ, Reiber JH, et al. Tumor diameter and volume assessed by magnetic resonance imaging in the prediction of outcome for invasive cervical cancer. Gynecol Oncol. 2001 Sep;82(3):474-82.

16. Iwata S, Joja I, Okuno K, Miyagi Y, Sakaguchi Y, Kudo T, et al. Cervical carcinoma with full-thickness stromal invasion: efficacy of dynamic MR imaging in the assessment of parametrial involvement. Radiat Med. 2002 Sep-Oct;20(5):247-55.

17. Pluta M, Rob L, Charvat M, Chmel R, Halaska M Jr, Skapa P, et al. Less radical surgery than radical hysterectomy in early stage cervical cancer: a pilot study. Gynecol Oncol.2009 May;113(2):1814. doi: 10.1016/j.ygyno.2009.02.005.

18. Frumovitz M, Sun CC, Schmeler KM, Deavers MT, Dos Reis R, Levenback CF, et al. Parametrial involvement in radical hysterectomy specimens for women with earlystage cervical cancer. Obstet Gynecol. 2009 Jul;114(1):93-9. doi: 10.1097/ AOG.0b013e3181ab474d.

19. Rob L, Pluta M, Strnad P, Hrehorcak M, Chmel R, Skapa P, et al. A less radical treatment option to the fertility-sparing radical trachelectomy in patients with stage I cervical cancer. Gynecol Oncol. 2008 Nov;111(2 Suppl):S116-20. doi: 10.1016/j.ygyno.2008.07.021.

20. Kim MK, Kim JW, Kim MA, Kim HS, Chung HH, Park NH, et al. Feasibility of less radical surgery for superficially invasive carcinoma of the cervix. Gynecol Oncol. 2010 Nov;119(2):18791. doi: 10.1016/j.ygyno.2010.06.027.

21. Wright JD, Grigsby PW, Brooks R, Powell MA, Gibb RK, Gao F, et al. Utility of parametrectomy for early stage cervical cancer treated with radical hysterectomy. Cancer. 2007 Sep 15;110(6):1281-6.

22. Kinney WK, Hodge DO, Egorshin EV, Ballard DJ, Podratz KC. Identification of a low-risk subset of patients with stage IB invasive squamous cancer of the cervix possibly suited to less radical surgical treatment. Gynecol Oncol. $1995 \mathrm{Apr} ; 57(1): 3-6$.

23. Stegeman M, Louwen $M$, van der Velden J, ten Kate FJ, den Bakker MA, Burger CW, et al. The incidence of parametrial tumor involvement in select patients with early cervix cancer is too low to justify parametrectomy. Gynecol Oncol. 2007 May;105(2):475-80.
24. Jung DC, Kim MK, Kang S, Seo SS, Cho JY, Park NH, et al. Identification of a patient group at low risk for parametrial invasion in early-stage cervical cancer. Gynecol Oncol. 2010 Dec;119(3):426-30. doi: 10.1016/j. ygyno.2010.08.005.

25. Chang SJ, Bristow RE, Ryu HS. A model for prediction of parametrial involvement and feasibility of less radical resection of parametrium in patients with FIGO stage IB1 cervical cancer. Gynecol Oncol. 2012 Jul;126(1):82-6. doi: 10.1016/j. ygyno.2012.04.016.

26. dos Reis R, Frumovitz M, Milam MR, Capp E, Sun CC, Coleman RL, et al. Adenosquamous carcinoma versus adenocarcinoma in early-stage cervical cancer patients undergoing radical hysterectomy: an outcomes analysis. Gynecol Oncol. 2007 Dec;107(3):45863.

27. Kodama J, Kusumoto T, Nakamura K, Seki N, Hongo A, Hiramatsu Y. Factors associated with parametrial involvement in stage IB1 cervical cancer and identification of patients suitable for less radical surgery. Gynecol Oncol. 2011 Sep;122(3):491-4. doi: 10.1016/j.ygyno.2011.05.038.

28. Puente R, Guzman S, Israel E, Poblete MT. Do the pelvic lymph nodes predict the parametrial status in cervical cancer stages IB-IIA? Int J Gynecol Cancer. 2004 Sep-Oct; $14(5): 832-40$.

29. Kamimori T, Sakamoto K, Fujiwara K, Umayahara K, Sugiyama Y, Utsugi $\mathrm{K}$, et al. Parametrial involvement in FIGO stage IB1 cervical carcinoma diagnostic impact of tumor diameter in preoperative magnetic resonance imaging. Int J Gynecol Cancer. 2011 Feb;21(2):349-54.

30. Gemer O, Eitan R, Gdalevich M, Mamanov A, Piura B, Rabinovich A, et al. Can parametrectomy be avoided in early cervical cancer? An algorithm for the identification of patients at low risk for parametrial involvement. Eur Jurnal Surg Oncol. 2013 Jan;39(1):76-80. doi: $10.1016 /$ j.ejso.2012.10.013.

Correspondencia: Carlos Urrutia Romero Dirección: Av. San Martin Mz-H3 Lt-12 Túpac Amaru, Chorrillos. Lima, Perú. Teléfono: 987418457

Correo electrónico:urrumed007@hotmail.com 